\title{
Guest Editorial: Special Issue on Testing of 3D Stacked Integrated Circuits
}

\author{
Erik Jan Marinissen • Yervant Zorian
}

Received: 17 January 2012 / Accepted: 17 January 2012 /Published online: 4 February 2012

(C) Springer Science+Business Media, LLC 2012

Three-dimensional stacked IC (3D-SIC) is a hot topic in both research and engineering circles alike. Multiple dies in a single package are not an entirely new phenomenon, as we already witnessed the use of Multi-Chip Modules (MCMs) two or three decades ago. We have even utilized vertical stacking of multiple chips, under terms as Package-onPackage (PoP) and System-in-Package (SiP). Recently however, a new and strong boost has been given to $3 \mathrm{D}$ die stacking with the advent of process technologies that enable the manufacturing of wafers containing Through-Silicon Vias (TSVs). TSVs allow architecting single-package multi-chip products using vertical interconnects with unprecedented density, performance, and low-power dissipation, thereby enabling the creation of a new generation of 'super chips'.

Test challenges are often considered as major obstacles to be resolved before bringing $3 \mathrm{D}$-SICs to market. The readers of JETTA probably know better than others that a semiconductor product is not ready for volume production, if it cannot be adequately tested for manufacturing defects. The fact that today many researchers and practitioners from the test community are working on these 3D-SIC test challenges is a positive sign indicating that the TSV-based stacking technology is preparing itself for prime-time. The level of professional activities has drastically increased, as we

\footnotetext{
E. J. Marinissen $(\square)$

IMEC,

Kapeldreef 75,

B-3001 Leuven, Belgium

e-mail: erik.jan.marinissen@imec.be

Y. Zorian

Synopsys,

700 East Middlefield Road,

Mountain View, CA 94043, USA

e-mail: yervant.zorian@synopsys.com
}

witnessed the increase from a single conference paper in 2007 to now numerous publications, conference sessions, tutorials, a thriving 3D test access architecture standardization working group: IEEE P1838 (http://grouper.ieee.org/ groups/3Dtest), and even a dedicated workshop: the IEEE International Workshop on Testing Three-Dimensional Stacked Integrated Circuits (http://3dtest.tttc-events.org). The 3D-TEST Workshop has already had two very successful editions in 2010 and 2011, and its third edition is scheduled for November 2012, again in conjunction with ITC.

What was so far still missing in this palette of activities was a journal special issue. That is now a reality with this February 2012 edition of JETTA. We find it quite appropriate for JETTA to be the first to publish a special issue on 3D-SIC testing, as after all this is the only journal devoted to test technology.

The open Call for Papers for this special issue was first published at the 3D-TEST Workshop 2010 with its submission deadline in January 2011. We received a good set of submissions, which were all subjected to a thorough peer review process with, in most cases, multiple iterations between authors and reviewers. The result is in front of you: a special issue with ten articles covering 3D-SIC testing from various angles.

This special issue starts off with a paper on test economics by Mottaqiallah Taouil et al. (Delft University of Technology and IMEC), entitled 'Test Impact on the Overall Dieto-Wafer 3D Stacked IC Cost'. The authors evaluate various test flows that include or exclude pre-, mid-, and post-bond tests with respect to their impact on overall product costs.

This is followed by two articles on defect-oriented testing. Yi Lou et al. (North-Carolina State University) compare sense amplification, leakage current monitors, and capacitance bridge methods as three pre-bond TSV test methods in 'Comparing Through-Silicon-Via (TSV) Void/Pinhole Defect 
Self-Test Methods'. In 'Fault Modeling and Multi-Tone Dither Scheme for Testing 3D TSV Defects', Sukeshwar Kannan et al. (University of Alabama and Yeungnam University) present a TSV test technique which uses an RF carrier signal modulated with a multi-tone signal with added Gaussian white noise to synthesize the test stimulus; the peak-to-average ratio is measured as output response.

The next two articles are on stress management, an important topic for 3D-SICs in which relatively large TSVs made out of copper or tungsten go through the thinneddown silicon substrate. Benjamin Backes et al. (University at Albany and Sematech) write in 'Effects of Copper Plasticity on the Induction of Stress in Silicon from Copper Through-Silicon Vias (TSVS) for 3D Integrated Circuits' about their finite-element modeling work to characterize the effect of copper elasto-plastic behavior on the induction of stress in 3D crystalline silicon systems with copper TSVs and the effect on TSV keep-out zones with respect to transistor placement. In 'Multi-Scale Simulation Methodology for Stress Assessment in 3D IC: Effect of Die Stacking on Device Performance', Valeriy Sukharev et al. (Mentor Graphics and Fraunhofer Institute for Non-Destructive Testing) present a set of compact models for accurate yet efficient multi-scale simulation of the mechanical stress across device layers in 3D-SICs.

The JETTA special issue continues with three papers on design-for-test (DfT). Erik Jan Marinissen et al. (IMEC, National Tsing-Hua University, and Delft University of Technology) describe in 'A DfT Architecture for 3D-SICs Based on a Standardizable Die Wrapper' a viable 3D DfT architecture, which involves a die wrapper that can be based on either IEEE Std 1149.1 or IEEE Std 1500 and enables a modular test approach. In 'On Testing Prebond Dies with Incomplete Clock Networks in a $3 D$ IC using DLLs', Michael Buttrick and Sandip Kundu (University of Massachusetts) propose the use of on-product clock generation with delay-locked loops (DLLs) to create a low-skew clock network in a pre-bond test setting. Brandon Noia et al. (Duke University and IMEC) present a unified framework to optimize the test architecture and corresponding test schedules for both (partial stack) mid-bond and (complete stack) post-bond testing in 'Optimization Methods for PostBond Testing of 3D Stacked ICs'.

Breeta SenGupta et al. (Linköping University) discuss test scheduling approaches to optimize the test application time for 3D-SICs in 'Scheduling Tests for 3D Stacked Chips under Power Constraints'. Finally, in 'CSL: Configurable Fault-Tolerant Serial Links for Inter-die Communication in $3 D$ Systems', Vladimir Pasca et al. (TIMA Laboratory) go beyond testing, by proposing a smart fault-tolerant infrastructure for TSV-based inter-die connections.
It is certainly not the case that this first special issue on 3D-SIC testing contains a final scientific and engineering verdict on all the test challenges in this field. To address new challenges as well as improve existing solutions, there is plenty of room for future publications, perhaps even for another special issue. But it is certainly an important milestone that the first special issue on this topic is now available!

Enjoy your reading.

Erik Jan Marinissen is Principal Scientist at IMEC vzw in Leuven, Belgium. Previously, he worked at NXP Semiconductors and Philips Research, both in Eindhoven, The Netherlands. Marinissen holds an MSc degree in Computing Science (1990) and a PDEng degree in Software Technology (1992), both from Eindhoven University of Technology. Marinissen's research interests include all topics in the domain of test and debug of micro-electronics. He is co-author of over 160 journal and conference papers and co-inventor on nine granted US and EP patent families. Marinissen is recipient of the ITC 2008 and ITC 2010 Most Significant Paper Awards and Best Paper Awards at the Chrysler-Delco-Ford Automotive Electronics Reliability Workshop 1995 and the IEEE International Board Test Workshop 2002. He served as Editor-in-Chief of IEEE Std. 1500. He is a founder of workshops on 'Diagnostic Services in Network-on-Chips' (DSNOC), '3D Integration', and 'Testing of Three-Dimensional Stacked Integrated Circuits' (3D-TEST). He serves on numerous conference committees, including ATS, DATE, ETS, ITC, and VTS, and on the editorial boards of IEEE Design \& Test of Computers, IET Computers and Digital Techniques, and Springer's Journal of Electronic Testing: Theory and Applications (JETTA). Marinissen is a Fellow of IEEE and Golden Core Member of Computer Society.

Yervant Zorian is a Chief Architect at Synopsys in Mountain View, California. Previously he was the Vice President and Chief Scientist of Virage Logic Corp, a Distinguished Member of Technical Staff at AT\&T Bell Laboratories, and Chief Technology Advisor of LogicVision Inc. Dr. Zorian received an MSc from the University of Southern California and a $\mathrm{PhD}$ from McGill University and an MBA from Wharton School of Business, University of Pennsylvania. He is currently the President of IEEE Test Technology Technical Council (TTTC), the Vice General Chair of Design Automation Conference (DAC), the Editor-in-Chief Emeritus of Design \& Test of Computers, the founder \& chair of IEEE 1500 Standardization Working Group, and an Adjunct Professor at the University of British Columbia. He served on the Board of Governors of Computer Society and CEDA, and as the Vice President of IEEE Computer Society. He has been founder and chair of a number of workshops and symposia, including the IEEE Workshops on 3D-IC Testing, Design-for-Manufacturability \& Yield, and East-West Design \& Test Symposium. Dr. Zorian holds 29 US patents, edited 4 books, authored over 300 refereed papers and received numerous best paper awards. He was selected by EE Times among the top 13 influencers on the semiconductor industry, was the 2005 recipient of the prestigious IEEE Industrial Pioneer Award for his contribution to BIST, and the 2006 recipient of the IEEE Hans Karlsson Award for diplomacy. A Fellow of the IEEE since 1999, he was recently elected a Foreign Member of the Academy of Sciences of Armenia. 